\title{
A STUDY OF DIASTOLIC DYSFUNCTION AND FACTORS INFLUENCING DIASTOLIC FUNCTION IN DIABETES MELLITUS PATIENTS IN CHENGALPATTU MEDICAL COLLEGE AND HOSPITAL
}

\author{
Velure Rajarao Mohan Rao1, Arjunan Senthil Kumaran², Subramanian Venkateswari ${ }^{3}$
}

1 Professor and HOD, Department of Medicine, Chengalpattu Medical College \& Hospital. ${ }^{2}$ Assistant Professor, Department of Medicine, Chengalpattu Medical College \& Hospital. 3Junior Resident, Department of Medicine, Chengalpattu Medical College \& Hospital.

\section{ABSTRACT}

A cross-sectional study on diastolic function and factors influencing diastolic function in diabetes mellitus patients with normal systolic function was done in Chengalpattu Medical College during the period of 2015-2016. All patients with type 2 DM of age 4080 years, duration of type 2 DM more than 5 years and with normal left ventricular systolic function was included in the study. Subjects with h/o angina, chest pain, ECG changes, abnormal treadmill test, evidence of valvular disease, hypertension and subjects with poor transthoracic ECHO window were excluded from study. The prevalence of diastolic dysfunction in asymptomatic type 2 DM patients is high and found to be $72 \%$ in the present study. The occurrence of diastolic dysfunction seems to precede the occurrence of systolic dysfunction in diabetes patients. There is a significant correlation between duration of diabetes, glycaemic control, age, obesity, dyslipidaemia and diastolic dysfunction among the seven parameters considered in the present study. Early diagnosis and initiation of appropriate treatment for diastolic dysfunction would slow down the progression to diastolic heart failure and cause significant reduction in the mortality and morbidity in patients with diabetes.

\section{KEYWORDS}

Diastolic Dysfunction, Dyslipidaemia, Left Ventricular Ejection Fraction, Glycaemic Control.

HOW TO CITE THIS ARTICLE: Rao VRM, Kumaran AS, Venkateswari S. A study of diastolic dysfunction and factors influencing diastolic function in diabetes mellitus patients in Chengalpattu Medical College and Hospital. J. Evolution Med. Dent. Sci. 2016;5 (70): 5058-5061, DOI: $10.14260 /$ jemds/2016/1149

\section{INTRODUCTION}

The disease burden of diabetes mellitus is increasing worldwide at a tremendous rate. Many studies have reported the occurrence of heart failure in diabetic patients is high even in the absence of coronary artery disease and hypertension. There is high prevalence of pre-clinical diastolic dysfunction as per the studies. Evidence suggests that diastolic dysfunction precedes systolic dysfunction in diabetic patients. So far only few population based studies have been conducted in India to demonstrate diastolic dysfunction in diabetic patients.

\section{MATERIAL AND METHODS}

The present study comprises of 100 patients of Diabetes mellitus of more than five-year duration, attending both Medical and Diabetology Outpatient Departments of Chengalpattu Government Medical College. This is a hospitalbased cross-sectional study involving rural and suburban population in and around Chengalpattu. All Type 2 Diabetes Mellitus patients of age 40 to 80 years of duration more than five years and with normal left ventricular systolic function were included in the study. Subjects with evidence of coronary artery disease, valvular heart diseases, systemic hypertension and patients with poor transthoracic Echo window were excluded from the study.

\section{RESULTS AND DISCUSSION}

Diabetes mellitus per se is a well-known independent risk factor for cardiovascular disease. The most common changes

Financial or Other, Competing Interest: None.

Submission 25-07-2016, Peer Review 19-08-2016,

Acceptance 24-08-2016, Published 31-08-2016.

Corresponding Author:

Velure Rajarao Mohan Rao,

Professor of Medicine,

Department of Medicine,

Chengalpattu Medical College.

E-mail: vrmohan_rao@yahoo.co.in

DOI: $10.14260 /$ jemds/2016/1149 in cardiovascular system associated with diabetes are left ventricular mass and structural changes, negative affection of the mid wall systolic mechanics and diastolic dysfunction.

Asymptomatic diastolic dysfunction is quite common among the population of type 2 diabetes mellitus patients. Various co-existing states like advancing age, obesity and dyslipidaemia and risk factors like smoking have both independent and additive effect over occurrence of diastolic dysfunction in the diabetes patients.

The asymptomatic diastolic dysfunction in diabetes precedes systolic dysfunction and is a common echocardiographic finding in diabetes patients with normal left ventricular systolic function.

Various diagnostic modalities like echocardiography, electrocardiogram and radionuclide scans which can be categorised as invasive and non-invasive can be utilised for identifying, assessing and quantifying the diabetes associated changes in cardiovascular system among which our study focuses on evaluating diastolic dysfunction using echocardiography.

Echocardiography has emerged as an excellent noninvasive diagnostic tool to delineate the details of the structure of the cardiac cavity, dimensions of cardiac walls and the wall movements. This is the reason why echocardiography is now widely used as a tool for assessing cardiac performance and cardiac structural abnormalities.

Left ventricular diastolic dysfunction has now emerged as a leading cause of cardiac morbidity in the Diabetes patients. The rate of association of defective diastolic performance, especially even in the asymptomatic stages is calculated to be high on the basis of available data.

Numerous parameters like myocardial compliance and relaxation, atrial contraction, pre-load, transvalvular pressure gradient, passive elastic properties, restraint of pericardium and the thoracic cage, respiratory variant, valve incompetence and arrhythmias influence the function of heart during diastolic phase. 
The development or progression of diastolic dysfunction can be discussed in mainly three stages, which are as follows: (1) Reduction in the early ventricular filling phase leading to inversion of ratio of peak early to peak atrial velocity curve, (2) Increased filling pressures in the atrial and ventricular chambers and reduction in ventricular relaxation causing Pseudo-normalisation of E/A flow pattern, (3) Restrictive pattern development due to various degrees of involvement of atrial flow.

Thus, a normal E/A flow pattern may be difficult to interpret as it may be associated with normal cardiac function or it may be associated with progressive diastolic cardiac dysfunction with raised filling pressures.

Takeda et al[1] by a study conducted in 544 Japanese DM patients with ejection fraction $\geq 50 \%$, found that diastolic dysfunction (impaired relaxation) plays a vital role in the induction of HF with normal systolic function in DM patients, regardless of the severity of DM and renal dysfunction.

Boyer et al[2] stated that the prevalence of LV diastolic dysfunction persons without symptoms and hypertension, but having type 2 diabetes disease is high. Diastolic dysfunction was found in $75 \%$ of the persons subjected to their study. In our study, prevalence of diastolic dysfunction was $72 \%$ in asymptomatic and normotensive patients out of the total 100 persons subjected to study.

In the current study, out of 100 patients who were subjected to the study $73 \%$ had poor glycaemic control and only $27 \%$ had good glycaemic control as measured using reliable parameters like $\mathrm{HbA1C}$.

Among the total 72 patients who had diastolic dysfunction, 64 patients which accounts for $87.7 \%$ in the total had poor glycaemic control. Also when assessed for co-relation between glycaemic control and diastolic dysfunction, the obtained value in the present study is ' $\mathrm{P}$ ' $<0.002$. This result is in concordance with study results of Soldatos et al,[3] who in their case control study of 55 individuals with type-2 DM concluded that diastolic dysfunction, present in a significant proportion of diabetic persons.

Van Heerebeek et al,[4] who conducted study in 36 type-2 DM patients concluded that the resting tension of cardiac myocyte is more reliable when LVEF is normal. Excessive diastolic left ventricular stiffness is an important contributor to heart failure in diabetic persons. Diabetes is presumed to increase stiffness through myocardial deposition of collagen and advanced glycation end products. Also in our current study, $72 \%$ of the diabetes patients were found to have diastolic dysfunction with normal LVEF.

Annonu et al[5] in their case control study of 66 subjects found that there was an inverse correlation between the duration of diabetes and E/A ratio $\left(\mathrm{r}=-0.4,{ }^{\prime} \mathrm{P}\right.$ '<.005). This result is comparable to our present study where among total 72 patients with diastolic dysfunction, $92.9 \%$ i.e. 26 of total 28 patients with duration of diabetes more than 10 years had diastolic dysfunction. Also in the total 72 patients with duration of diabetes of 5 - 10 years, 46 patients i.e. $63.9 \%$ had diastolic dysfunction. Also the correlation between duration of diabetes and diastolic dysfunction was calculated in present study as ' $\mathrm{P}$ ' $<0.004$.

Masugata et $\mathrm{al},[6]$ who conducted a case control study in 77 persons with no hypertension patients found that the cardiac diastolic dysfunction without LV systolic dysfunction in patients with type $2 \mathrm{DM}$ with good glycaemic control is related neither to LV hypertrophy nor to hypertension, but rather to aging and the duration of type 2 DM. Similarly, in our current study, $72 \%$ of total subjects without hypertension and CAD had diastolic dysfunction with normal left ventricular systolic function. In their study of 484 subjects between 1996 to 2007 year found that a duration of diabetes $\geq 4$ years was independently associated with $\mathrm{LV}$ diastolic dysfunction (E/e' $>15$ ) with odds ratio 1.91 .

Mishra et al[7] in their case control study of 71 subjects with type $2 \mathrm{DM}$ found that asymptomatic diabetic patients have reduced LV systolic and diastolic function as compared with healthy subjects.

In the current study, out of 100 patients 51 belonged to age group of 40-50 years and among them 29 persons that is $56.9 \%$ had diastolic dysfunction. In the age group of 51-60 years, 48 persons were studied and among them 42 persons that is $87.5 \%$ were evaluated to have diastolic dysfunction. As the number of patients studied above this age group were meagre, the results could not be extended.

With the available data from present study, it is seen that there is strong co-relation between age and diastolic dysfunction with a value of ' $\mathrm{P}$ ' $<0.003$. Whether this correlation could be solely attributed to age factor or due to additive effect of increase in the duration of diabetes with advancing age could not be delineated by the present study as all cases studied were diabetes patients.

So far various studies have shown various degrees of association between gender and diastolic dysfunction. In the present study, out of total subjects $72 \%$ had diastolic dysfunction. Among them 55.6\% were females and $44.4 \%$ were males. When calculated for correlation between gender and diastolic dysfunction, the obtained value ' $\mathrm{P}$ ' $<0.254$. Thus, present study does not show a strong association between gender and diastolic dysfunction.

According to present study, out of total subjects $69 \%$ were obese and $31 \%$ were not obese. Among the obese individuals who participated in the present study, $81.2 \%$ had diastolic dysfunction. Out of total 72 individuals who had diastolic dysfunction $77.8 \%$ that is 56 persons come under obese category, whereas $22.2 \%$ that is 16 persons come under nonobese category. When calculated for correlation between obesity and diastolic dysfunction, the obtained value is ' $\mathrm{P}$ ' $<0.002$ showing strong correlation between obesity and diastolic dysfunction.

Peterson et al[8] designed a study to determine the effects of obesity on LV structure and function in obese individuals. Fifty-one subjects were evaluated and among them 20 were obese having BMI $\geq 30 \mathrm{~kg} / \mathrm{m} 2$ and their $\mathrm{LV}$ structure and diastolic function was evaluated by $2 \mathrm{D}$-echo. The result showed that subjects who were obese had higher and septal and posterior wall thickness during diastolic phase.

Grandi et al[9] evaluated the influence of obesity on LV diastolic function. They selected 32 non-obese, 32 obese normotensives matched for age, sex and BMI. Results showed the main effect was found for obesity on LV diameter and LV mass, LV systolic function was normal in all the subjects and LV diastolic function was significantly reduced. They concluded that obesity is associated with a preclinical impairment of LV diastolic function.

Results from the above studies are in concordance of those of our present study, which too shows a significant correlation between obesity and diastolic dysfunction. 
From the data available from the present study, out of total 72 individuals who had diastolic dysfunction $87.5 \%$ that is 63 persons had dyslipidaemia and $12.5 \%$ that is 9 persons were found to have normal lipid profile. When calculated for association between dyslipidaemia and diastolic dysfunction, the obtained value is ' $\mathrm{P}$ ' $<0.05$ showing a strong co-relation between dyslipidaemia and diastolic dysfunction.

Fukuta et al[10] studied the correlation between dyslipidaemia and diastolic dysfunction in 45 age and sex matched cases and controls with dyslipidaemia and normal lipid profile and found that there is high association between dyslipidaemia and diastolic dysfunction. Result from this study is in concordance of those of our present study which also shows a significant correlation between obesity and diastolic dysfunction.

Garcia et al[11] conducted a study, wherein 78 matched cases who were smokers and 78 controls who were nonsmokers were included. Cardiac performance of all the subjects was assessed using standardised echocardiographic parameters.

Results of the above study showed there is decline in the cardiac performance in the case group when compared to the control group as evident by increase in left ventricular mass and stiffness and poor wall compliance leading to diastolic dysfunction.

In the present study, out of 100 subjects studied only $26 \%$ were smokers and the remaining 74\% were non-smokers. Among the 72 individuals who were found to have diastolic dysfunction by echocardiographic parameters, 18 persons that is $25 \%$ were smokers and 54 persons that is $75 \%$ were non-smokers.

When calculated for association between smoking and diastolic dysfunction, the value obtained is ' $\mathrm{P}$ ' $>0.05$ showing no significant correlation between these cause and effect. Many reasons may be attributed for this result.

Among the total study population, the number of subjects with history of smoking was only $26 \%$. Gender distribution with a female preponderance among the study population may also contribute as in a country like India the habit of smoking among the female population is low when compared to other nations. All these factors may have led to the negative correlation between smoking and diastolic dysfunction in the present.

\section{DIASTOLICDYSFUNCTION AND GLYCAEMIC STATUS}

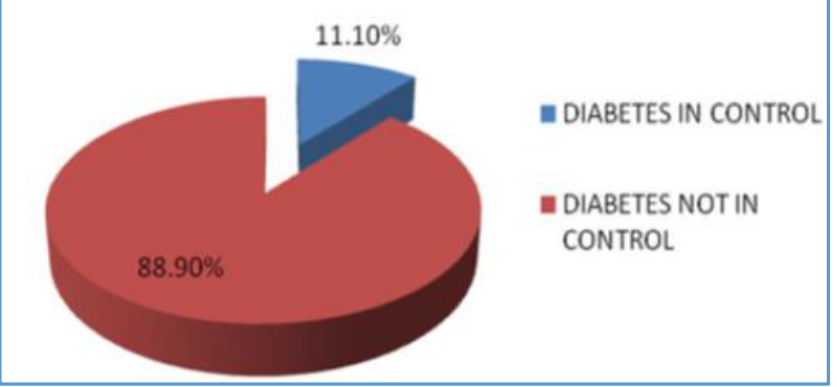

Fig. 1: Showing Diastolic Dysfunction and Glycaemic Status

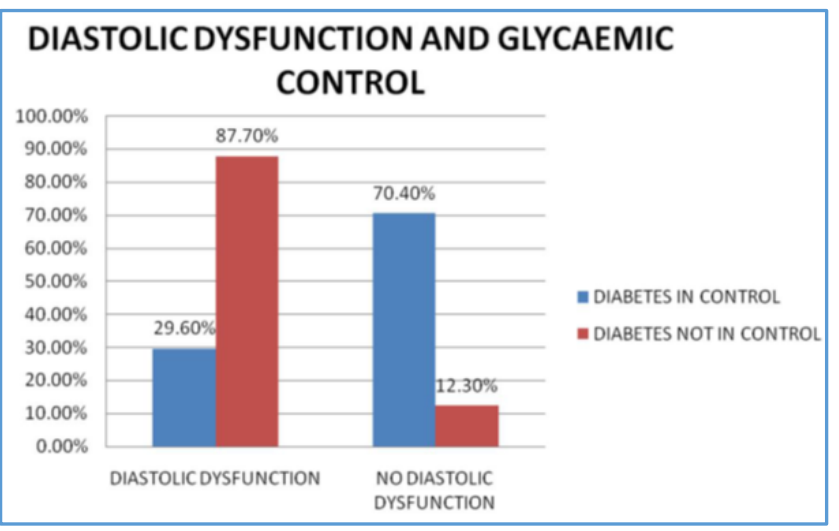

Fig. 2: Showing Correlation between Diastolic Dysfunction and Glycaemic Control

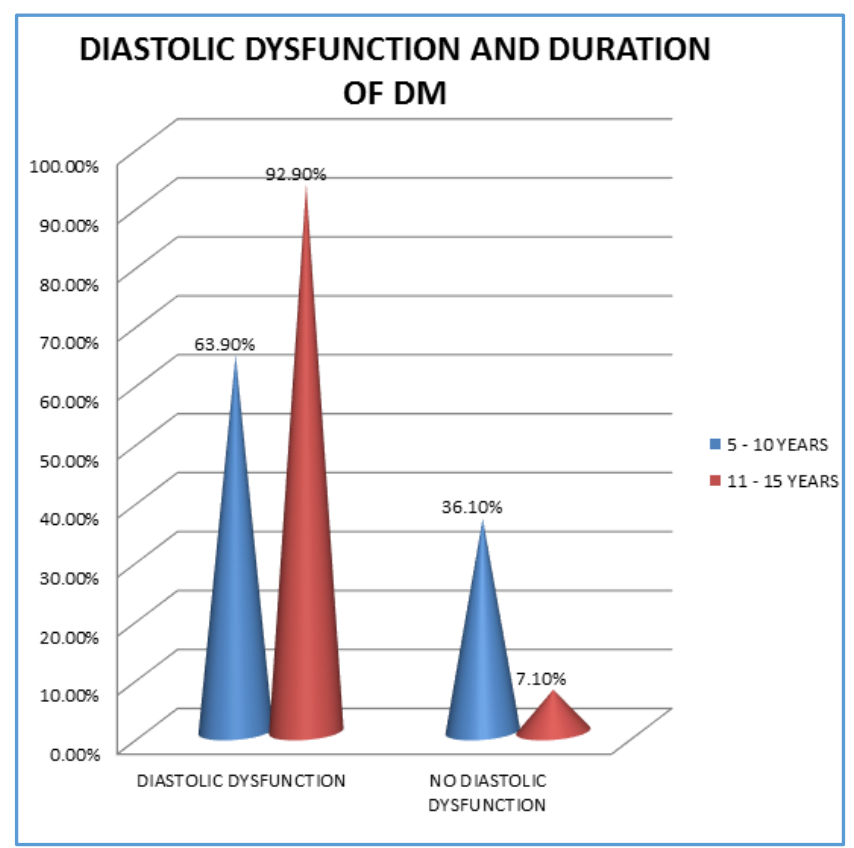

Fig. 3: Showing Correlation between Diastolic Dysfunction and Duration of Diabetes

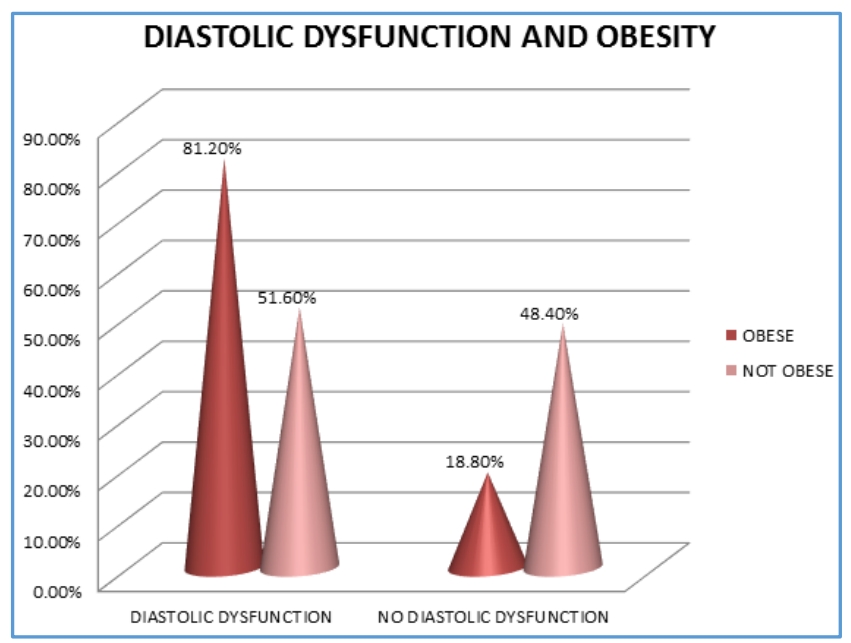

Fig. 4: Showing Correlation between Diastolic Dysfunction and Obesity 
CORRELATION BETWEEN GENDER AND DIASTOLIC DYSFUNCTION

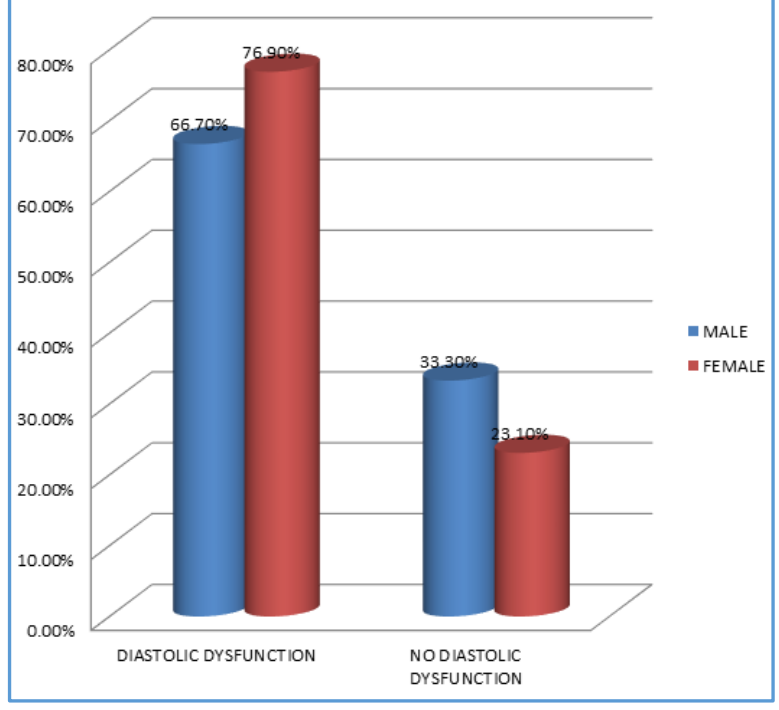

Fig. 5: Showing Correlation between Gender and Diastolic Dysfunction

\begin{tabular}{|c|c|c|c|}
\hline \multirow{2}{*}{ Obesity } & \multicolumn{3}{|c|}{ Normal Diastolic Function } \\
\cline { 2 - 4 } & Number & $\begin{array}{c}\text { \% in } \\
\text { Obesity }\end{array}$ & $\begin{array}{c}\text { \% in Normal } \\
\text { Diastolic Function }\end{array}$ \\
\hline OBESE & 13 & $18.8 \%$ & $46.4 \%$ \\
\hline $\begin{array}{c}\text { NON- } \\
\text { OBESE }\end{array}$ & 15 & $48.4 \%$ & $53.6 \%$ \\
\hline Table 1: Showing Correlation between Normal Diastolic \\
Function and Obesity \\
\hline
\end{tabular}

\begin{tabular}{|c|c|c|c|}
\hline \multirow{2}{*}{ Obesity } & \multicolumn{3}{|c|}{ Diastolic Dysfunction } \\
\cline { 2 - 4 } & Number & $\begin{array}{c}\text { \% in } \\
\text { Obese }\end{array}$ & $\begin{array}{c}\text { \% in Diastolic } \\
\text { Dysfunction }\end{array}$ \\
\hline OBESE & 56 & $81.2 \%$ & $77.8 \%$ \\
\hline $\begin{array}{c}\text { NON- } \\
\text { OBESE }\end{array}$ & 16 & $51.6 \%$ & $22.2 \%$ \\
\hline \multicolumn{3}{|c|}{ Table 2: Showing Correlation between Diastolic } \\
Dysfunction and Obesity
\end{tabular}

\begin{tabular}{|c|c|c|c|}
\hline \multirow{2}{*}{ Lipid Profile } & \multicolumn{3}{|c|}{ Diastolic Dysfunction } \\
\cline { 2 - 4 } & Number & $\begin{array}{c}\text { \% in } \\
\text { Lipid } \\
\text { Profile }\end{array}$ & $\begin{array}{c}\text { \% in Diastolic } \\
\text { Dysfunction }\end{array}$ \\
\hline DYSLIPIDAEMIA & 63 & $87.5 \%$ & $87.5 \%$ \\
\hline NO & 9 & $32.1 \%$ & $12.5 \%$ \\
DYSLIPIDAEMIA & 9 & \multicolumn{3}{|c|}{ Table 3: Showing Correlation between Diastolic } \\
Dysfunction and Dyslipidaemia
\end{tabular}

\begin{tabular}{|c|c|c|c|}
\hline \multirow{2}{*}{ Lipid Profile } & \multicolumn{3}{|c|}{ Normal Diastolic Function } \\
\cline { 2 - 4 } & No. & $\begin{array}{c}\text { \% in } \\
\text { Dyslipidaemia }\end{array}$ & $\begin{array}{c}\text { \% in } \\
\text { Normal } \\
\text { Diastolic } \\
\text { Function }\end{array}$ \\
\hline DYSLIPIDAEMIA & 9 & $12.5 \%$ & $32.1 \%$ \\
\hline $\begin{array}{c}\text { NO } \\
\text { DYSLIPIDAEMIA }\end{array}$ & 19 & $67.9 \%$ & $67.9 \%$ \\
\hline $\begin{array}{c}\text { Table 4: Showing Co-relation between Normal Diastolic } \\
\text { Function and Dyslipidaemia }\end{array}$ \\
\hline
\end{tabular}

\section{CONCLUSION}

The prevalence of diastolic dysfunction in asymptomatic type $2 \mathrm{DM}$ patients is high and found to be $72 \%$ in the present study.

The occurrence of diastolic dysfunction seems to precede the occurrence of systolic dysfunction in diabetes patients.

There is a significant correlation between duration of diabetes, glycaemic control, age, obesity, dyslipidaemia and diastolic dysfunction among the seven parameters considered in the present study.

Persons with type 2 diabetes mellitus should be screened with echocardiography for subclinical diastolic dysfunction.

Early diagnosis and initiation of appropriate treatment for diastolic dysfunction would slow down the progression to diastolic heart failure and cause significant reduction in the morbidity and mortality in patients with diabetes.

\section{REFERENCES}

1. Takeda Y, Sakata Y, Mano T, et al. Competing risks of heart failure with preserved ejection fraction in diabetic patients. Eur J Heart Fail 2011;13(6):664-9.

2. Boyer JK, Thanigaraj S, Schechtman KB, et al. Prevalence of ventricular diastolic dysfunction in asymptomatic, normotensive patients with diabetes mellitus. Am J Cardiol 2004;93(7):870-5.

3. Soldatos G, Jandeleit-Dahm K, Thomson H, et al. Large artery biomechanics and diastolic dysfunction in patients with type 2 diabetes. Diabet Med 2011;28(1):54-60.

4. Van Heerebeek L, Hamdani N, Handoko ML, et al. Diastolic stiffness of the failing heart: importance of fibrosis, advanced Glycation end products and myocyte resting tension. Circulation 2008;117(1):43-51.

5. Annonu AK, Fattah AA, Mokhtar MS, et al. Left ventricular systolic and diastolic functional abnormalities in asymptomatic patients with non-insulin-dependent diabetes mellitus. J Am Soc Echocardiogr 2001;14(9):885-91.

6. Masugata $H$, Senda $S$, Goda $F$, et al. Left ventricular diastolic dysfunction in normotensive diabetic patients in various age strata. Diabetes Res Clin Pract 2008;79(1):91-6.

7. Mishra TK, Rath PK, Mohanty NK, et al. Left ventricular systolic and diastolic dysfunction and their relationship with microvascular complications in normotensive, asymptomatic patients with type 2 diabetes mellitus. Indian Heart J 2008;60(6):548-53.

8. Peterson LR, Waggoner AD, Schechtman KB, et al. Alterations in left ventricular structure and function in young healthy obese women: assessment by echocardiography and tissue Doppler imaging. J Am Coll Cardiol 2004;43(8):1399-404.

9. Grandi AM, Zanzi P, Piantanida E, et al. Obesity and left ventricular diastolic function: non-invasive study in normotensives and newly diagnosed never-treated hypertensives. Int J Obes 2000;24(8):954-8.

10. Fukuta H, Ohte N, Wakami K, et al. Prognostic value of left ventricular diastolic dysfunction in patients undergoing cardiac catheterization for coronary artery disease. Cardiol Res Pract 2012;2012:243735.

11. Garcia MJ, Thomas JD, Klein AL. New Doppler echocardiographic applications for the study of diastolic function. Journal of the American College of Cardiology 1998;32(4):865-75. 